\title{
FLUCTUATIONS ANNUELLES DES POPULATIONS DE PHLÉbotomes (DIPTERA, PHLEBOTOMIDAE) dans la province de Grenade (Espagne)
}

\author{
F. MORILLAS MARQUEZ*, D. C. GUEVARA BENITEZ**, \\ J. M. UBEDA ONTIVEROS** et J. GONZALEZ CASTRO*
}

RÉSUMÉ. Les fluctuations saisonnières des populations de phlébotomes présentes dans la province de Grenade (Espagne) ont été étudiées sur une période d'un an par la méthode de capture aux pièges adhésifs.

Parmi les espèces récoltées seules $S$. minuta, $P$. perniciosus et $P$. ariasi ont été assez abondantes pour permettre des études quantitatives.

$S$. minuta évolue de façon monophasique, avec un maximum d'activité durant les mois chauds (juillet, août, septembre).

En revanche $P$. perniciosus et $P$. ariasi ont une évolution biphasique avec un maximum de captures au début et un autre à la fin de la saison chaude.

\section{Annual fluctuations of Phlebotomidae populations in the province of Granada (Spain)}

SUMMARY. During a whole year, in order to know the annual evolution of the populations, a sampling of sandflies fauna in Granada (Spain) was carried out. The method of capture used was paper oiled.

From the collected species, only $S$. minuta, $P$. perniciosus and $P$. ariasi were present in sufficient numbers to enable a proper observation of evolution. The first of these follows a monophasic course with maximum activity during the warmest months (July, August, September). However, $P$. perniciosus and $P$. ariasi show, in general, a diphasic curve with a highest rate at the beginning and end the warm season.

\section{Introduction}

Les travaux ayant trait aux phlébotomes d'Espagne ont été surtout consacrés jusqu'à présent à des inventaires qualitatifs et quantitatifs (Pittaluga et De Buen, 1918 ; Najera Angulo, 1940 ; Zariquiey Alvarez, 1944 ; Houin, 1965 ; Guevara Benitez

\footnotetext{
* Dpt. Parasitologie, Fac. Pharmacie, Grenade. Espagne.

** Dpt. Parasitologie, Fac. Pharmacie, Sevilla. Espagne.

Accepté le II mars 1983 .
} 
et coll., 1978) et à des études éthologiques en rapport notamment avec l'anthropophilie et la zoophilie des diverses espèces (Elvira, 1931 ; Gil Collado, 1931 ; Vives Sabater, 1954 ; Martinez Ortega et coll., 1982). Aucune enquête systématique sur les variations saisonnières des populations n'avait encore été réalisée.

Nous rapportons ici les résultats de captures effectuées au cours d'une même année, à intervalles réguliers, dans la province de Grenade dans laquelle plusieurs cas de leishmaniose viscérale et cutanée sont signalés chaque année.

\section{Matériel et méthodes}

La méthode de capture utilisée est celle des pièges adhésifs, particulièrement bien adapté à l'inventaire des gîtes de repos des phlébotomes dans une région étendue (Rioux et coll., 1967). Cette méthode ne nécessite pas une surveillance des pièges et élimine les causes de variations inhérentes aux conditions météorologiques.

Les pièges sont constitués par des feuilles de papier de $31 \times 21 \mathrm{~cm}, 21 \times 16 \mathrm{~cm}$ et $16 \times 10 \mathrm{~cm}$, imprégnées d'huile de ricin.

Ils sont maintenus en place pendant 15 jours et remplacés au bout de ce temps par des pièges neufs. Vingt-six piégeages successifs ont été ainsi réalisés. Pour obtenir une exploration aussi étendue que possible de la zone d'endémie leishmanienne, les stations de piégeage ont été choisies le long de quatre itinéraires, certaines au voisinage des habitations humaines, d'autres en pleine nature, en accord avec les conditions de terrain, en des points distants de 8 à $10 \mathrm{~km}$.

Le tracé de ces itinéraires est le suivant (fig. 1) :

- Itinéraire I. - Grenade-Loja. Route nationale 342. Trajet de $60 \mathrm{~km}$ avec $7 \mathrm{sta}-$ tions dans les localités de Grenade (quartier de la Chana), Chauchina, Lachar, Fuensanta, Moraleda Zafayona, Salar et Loja. C'est une zone de climat méditerranéen avec des variations saisonnières bien marquées.

- Itinéraire II. - Grenade-Guadix. Route nationale 342. Trajet de $60 \mathrm{~km}$ avec des stations à Grenade (Haza Grande), Huetor Santillan, La Peza, Diezma et Graena. Le climat de cette région peut être considéré comme continental.

- Itinéraire III. - Venta de las Angustias-Albuñol. Route départementale 333. Trajet de $60 \mathrm{~km}$ avec 7 lieux de piégeage : Lanjarón (2), Orgiva, Alcazar, Polopos, Sorvilán et Albuñol. Le climat méditerranéen y est plus modéré que le long de l'itinéraire I.

- Itinéraire IV. - Route nationale 340, le long de la côte, de La Rábita à Almuñecar. Il comporte 6 stations situées dans les localités de : La Rábita, La Mamola, Castelldelferro, Motril, Salobreña et Almuñecar.

\section{Résultats}

Sept espèces ont été récoltées : Sergentomyia minuta (Rondani, 1843) (9 197 exemplaires), Phlebotomus perniciosus Newstead, 1911 (3 620 exemplaires), P. ariasi Tonnoir, 1921 (1 185 exemplaires), P. longicuspis (Nitzulescu, 1930) (15 exemplaires), 


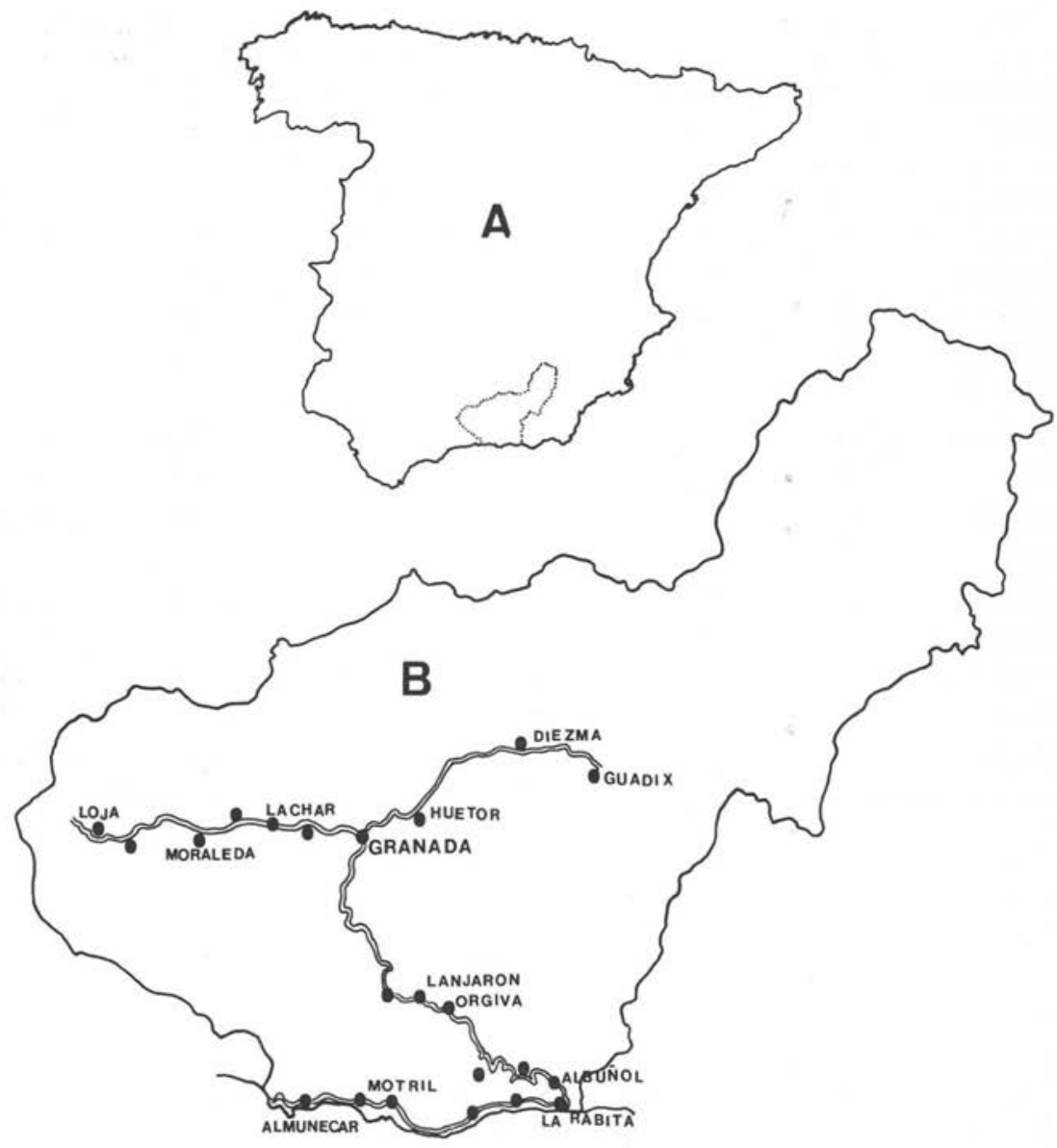

FIg. I. - A) Carte de l'Espagne indiquant la province de Grenade, où se sont réalisés les investigations.

B) Carte de Grenade montrant les lieux où se sont faits les captures. E. : r.ooo.ooo.

P. sergenti Parrot, 1917 (137 exemplaires), P. chabaudi Croset, Abonnenc et Rioux, 1970 (4 exemplaires) et $P$. papatasi (Scopoli, 1786) (29 exemplaires).

Le tableau $I$ donne l'analyse par espèces ( $\widehat{\jmath}$ et ( ) de nos captures. Il met en évidence la prédominance de $S$. minuta, $P$. perniciosus et $P$. ariasi par rapport aux autres espèces.

- Sergentomyia minuta (Rondani, 1843) est l'espèce la plus abondante, avec une période d'activité qui s'étend du 20 mars (1 ${ }^{\text {res }}$ captures) au 23 décembre (dernières captures). La figure 2 montre, pour les itinéraires I, II et III, une évolution des effectifs qui peut être considérée comme monophasique avec un seul maximum important 
Tableau I. - Numéros de Phlébotomes capturés pour chaque itinéraire.

Les résultats sont exprimés en valeurs absolues. $m=$ mâles; $f=$ femelles.

\begin{tabular}{|c|c|c|c|c|c|c|}
\hline Especies & Sexo & I & II & III & IV & Total \\
\hline $\begin{array}{l}\text { Sergentomyia } \\
\text { minuta }\end{array}$ & $\begin{array}{c}\mathrm{m} \\
\mathrm{f}\end{array}$ & $\begin{array}{l}1675 \\
\mathrm{I} 128\end{array}$ & $\begin{array}{l}794 \\
534\end{array}$ & $\begin{array}{l}2385 \\
1727\end{array}$ & $\begin{array}{l}5^{18} \\
43^{6}\end{array}$ & $\begin{array}{l}5372 \\
3825\end{array}$ \\
\hline $\begin{array}{l}\text { Phlebotomus } \\
\text { perniciosus }\end{array}$ & $\underset{f}{m}$ & $\begin{array}{r}\text { I } 544 \\
\text { I } 80\end{array}$ & $\begin{array}{r}326 \\
46\end{array}$ & $\begin{array}{r}1109 \\
132\end{array}$ & $\begin{array}{r}243 \\
40\end{array}$ & $\begin{array}{r}3222 \\
398\end{array}$ \\
\hline $\begin{array}{r}\text { Phlebotomus } \\
\text { longicuspis }\end{array}$ & $\begin{array}{c}\mathrm{m} \\
\mathrm{f}\end{array}$ & ${ }^{6}$ & - & ${ }^{8}$ & $-^{\mathbf{I}}$ & $-^{I_{5}}$ \\
\hline $\begin{array}{l}\text { Phlebotomus } \\
\text { ariasi }\end{array}$ & $\begin{array}{c}\mathrm{m} \\
\mathrm{f}\end{array}$ & $\begin{array}{r}570 \\
4^{\mathrm{I}}\end{array}$ & $\begin{array}{r}32 \\
6\end{array}$ & $\begin{array}{r}362 \\
48\end{array}$ & $\begin{array}{r}120 \\
8\end{array}$ & $\begin{array}{r}1084 \\
\text { 103 }\end{array}$ \\
\hline $\begin{array}{l}\text { Phlebotomus } \\
\text { sergenti }\end{array}$ & $\begin{array}{c}\mathrm{m} \\
\mathrm{f}\end{array}$ & $\begin{array}{l}36 \\
17\end{array}$ & $-^{2}$ & $\begin{array}{l}47 \\
\text { II }\end{array}$ & $\begin{array}{r}20 \\
4\end{array}$ & $\begin{array}{r}105 \\
32\end{array}$ \\
\hline $\begin{array}{l}\text { Phlebotomus } \\
\text { chabaudi }\end{array}$ & $\begin{array}{c}\mathrm{m} \\
\mathrm{f}\end{array}$ & - & - & - & $-^{4}$ & $-^{4}$ \\
\hline $\begin{array}{l}\text { Phlebotomus } \\
\text { papatasi }\end{array}$ & $\begin{array}{l}\mathrm{m} \\
\mathrm{f}\end{array}$ & $-^{2}$ & - & $\begin{array}{l}6 \\
3\end{array}$ & $\begin{array}{r}16 \\
2\end{array}$ & $\begin{array}{r}26 \\
5\end{array}$ \\
\hline TOTAL & & 5199 & I740 & $5^{8} 3^{8}$ & I $4 I_{2}$ & I 4189 \\
\hline
\end{tabular}

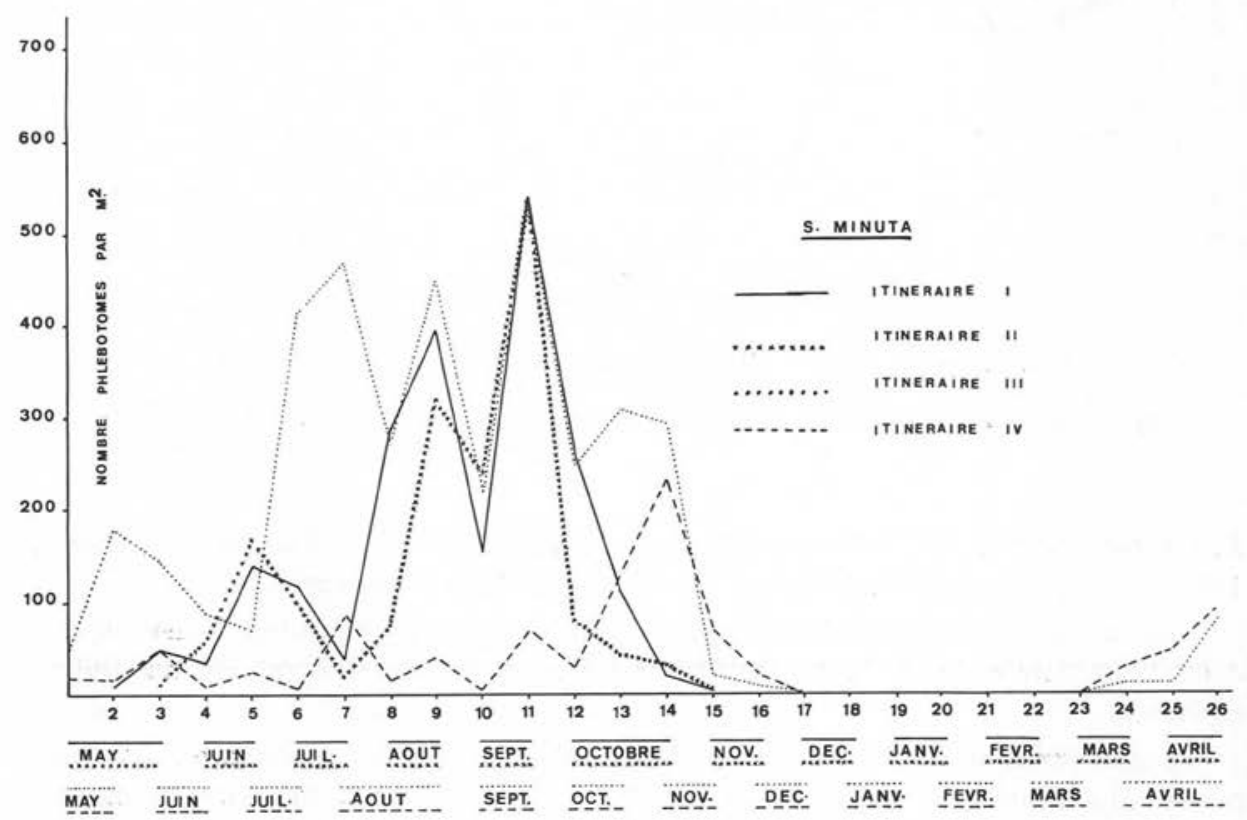

FIG. 2. - Fluctuations annuelles de Sergentomyia minuta (Rondani, I843) dans les quatre itinéraires d'échantillonnage. 
pour la période juillet-septembre. La courbe correspondant à l'itinéraire IV, le long duquel le nombre des captures a été très inférieur, est nettement différente. Elle montre une succession de clochers et des creux qui semble n'obéir à aucune règle.

- Phlebotomus perniciosus Newstead, 1911 (fig. 3) possède une période d'activité qui va de mai à décembre pour les itinéraires I et III, avec une courbe nettement diphasique. On observe un premier maximum de la mi-juin à la fin juillet et un second fin septembre-fin octobre. Le nombre des captures diminue, sans s'annuler durant la période la plus chaude de l'année.

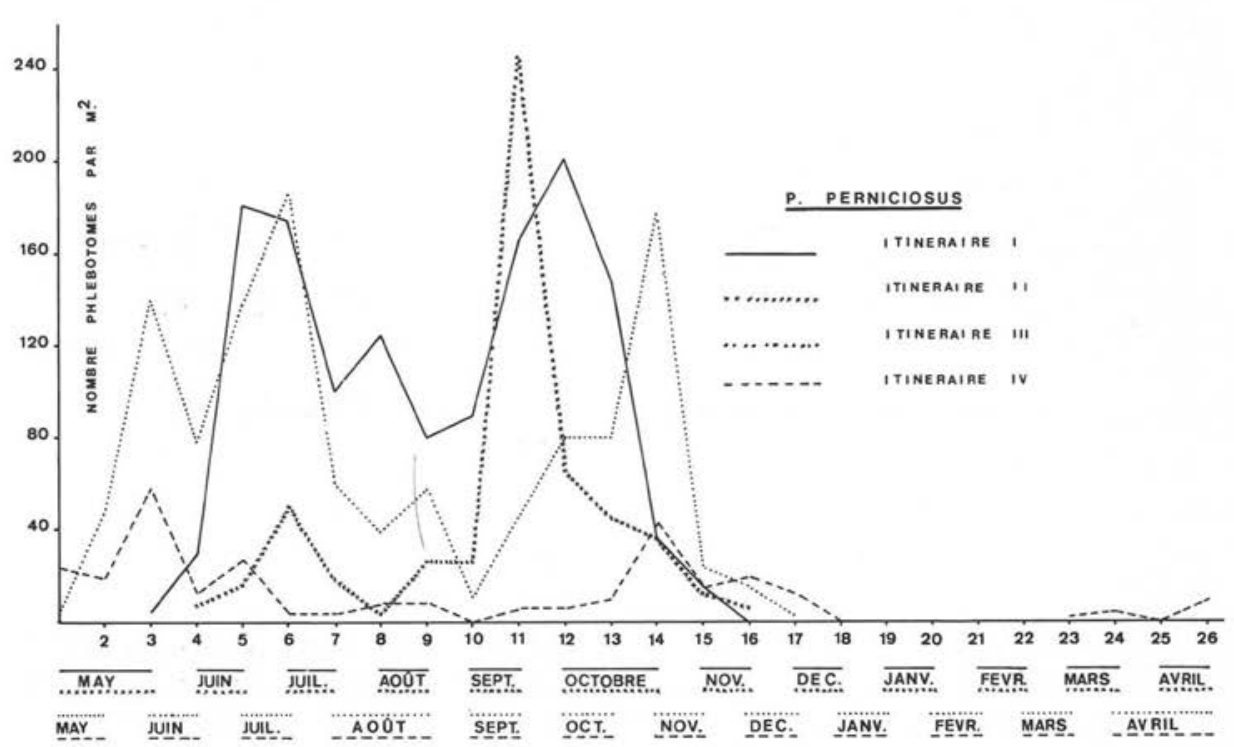

Fig. 3. - Fluctuations annuelles de Phlebotomus perniciosus Newstead, I9I I dans les quatre itinéraires d'échantillonnage.

Pour l'itinéraire II, la période d'activité est plus courte (juillet-novembre) et le maximum se situe à la fin de l'été. Pour l'itinéraire IV au contraire, la période d'activité est plus longue (20 mars-23 décembre) et les variations peu marquées, si ce n'est qu'on observe une légère augmentation au début juin et une autre en novembre.

- Phlebotomus ariasi Tonnoir, 1921, montre une évolution assez parallèle à celle de P. perniciosus ( fig. 4). Pour les itinéraires I et III, les captures ont été effectuées entre mars et décembre et la courbe est diphasique avec un maximum en mai-juin et un autre en septembre-octobre-début novembre. On observe une chute importante durant l'été ( $2^{\mathrm{e}}$ moitié de juillet-août-début septembre).

Pour l'itinéraire IV, le nombre de captures est resté constamment bas, mais l'activité de l'espèce persiste toute l'année, y compris les mois de janvier et février. 


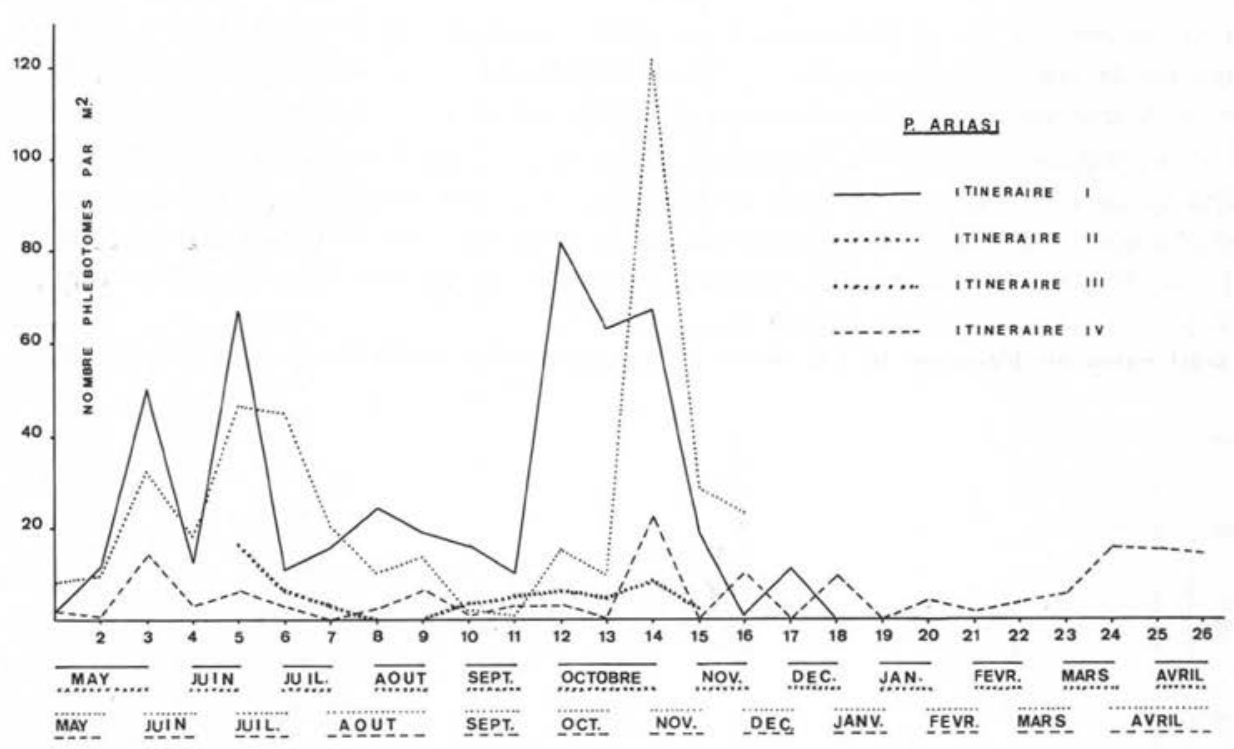

FIG. 4. - Fluctuations annuelles de Phlebotomus ariasi Tonnoir I92I dans les quatre itinéraires.

\section{Discussion}

Nos résultats sont généralement en accord avec ceux rapportés par d'autres auteurs de régions voisines de l'Espagne où les mêmes espèces sont rencontrées. Cependant il convient de noter quelques différences. La courbe monophasique obtenue avec $S$. minuta pour les itinéraires I, II et III, est tout à fait comparable à celle trouvée par Croset (1969) dans le sud de la France et en Tunisie. Il en est de même pour la courbe diphasique obtenue avec $P$. perniciosus pour les itinéraires I et III. Cependant nous avons obtenu une courbe monophasique pour l'itinéraire II. D'interprétation difficile, cette particularité est peut être liée au climat nettement continental qui caractérise cette région.

$P$. ariasi montre à Grenade une évolution diphasique différente de celle observée dans le sud de la France. Cette différence de comportement est peut-être à mettre sur le compte des conditions climatiques rencontrées en France (températures moins élevées, saison chaude plus courte) ou sur celui des facteurs écologiques propres à l'une et l'autre de ces régions. La durée des périodes d'activité diffère aussi notablement. Alors que dans le sud de la France celles de $S$. minuta et de $P$. perniciosus est comprise entre juin et octobre, nos captures s'échelonnent du 20 mars au 23 décembre. A noter que les sorties de $S$. minuta parroti en Tunisie ont lieu de la fin du mois d'avril au 15 novembre et que celles de $S$. minuta en Italie ont été signalées du 25 juin au 22 septembre (Maroli et Bettini, 1977). 
Il en est de même pour $P$. perniciosus : 24 avril-19 décembre en Tunisie, juinseptembre en France, 9 juin-22 octobre en Italie.

Les différences sont encore plus marquées pour $P$. ariasi. Nous avons capturé cette espèce toute l'année le long de l'itinéraire IV (climat subtropical) et seulement de mars à décembre sur les itinéraires I et III. En France la période d'activité ne dure que de mai à novembre.

$P$. sergenti, $P$. papatasi, $P$. longicuspis et $P$. chabaudi ont été capturés en trop petit nombre pour permettre l'étude de leur cycle annuel. Les deux dernières espèces sont rares en Espagne $(0,1 \%$ pour $P$. longicuspis et $0,028 \%$ pour $P$. chabaudi) et de découverte récente (Morillas Marquez et coll., 1982 ; Rioux et coll., 1974).

$P$. sergenti, capturé de mai à décembre, et $P$. papatasi, étant deux espèces anthropophiles et endophiles, sont peu représentées dans nos piégeages qui ont été réalisés à l'extérieur.

\section{Conclusions}

Pour S. minuta la période d'activité a durée du 20 mars au 23 décembre. L'évolution est monophasique et la densité est maximum en juillet, août et septembre. Avec $P$. perniciosus et $P$. ariasi, la courbe d'évolution est diphasique pour les itinéraires I et III et présente deux maxima au début et à la fin de la saison chaude. Sur l'itinéraire IV, $P$. ariasi est présent durant toute l'année, y compris les mois de janvier et février.

\section{BIBLIOGRAPHIE}

Bailly-Choumara H., Abonnenc E., Pastre J. : Contribution à l'étude des Phlébotomes du Maroc (Diptera, Psychodidae). Données faunistiques et écologiques. Cah. ORSTOM, ser. ent. Med. Parasitol., I97I, 9, 43I-460.

Croset H. : Écologie et Systématique des Phlebotomini (Diptera, Psychodidae) dans deux foyers, français et tunisien, de Leishmanioses viscérales. Essai d'interprétation épidémiologique. Thèse Sciences, Montpellier, 1969, $516 \mathrm{p}$.

Croset H., Rioux J. A., Juminer B., Tour S. : Fluctuations annuelles des populations de Phlebotomus perniciosus Newstead, I9II, Phlebotomus perfiliewi Parrot, I930 et Sergentomyia minuta parroti (Adler et Theodor, 1927) (Diptera, Psychodidae) en Tunisie du Nord. Arch. Inst. Pasteur Tunis, I970, 47, 43-56.

Elvira J. : Nota sobre la biología de Phlebotomus perniciosus. Med. Pais. Cál., 1931, 4, 52-53.

FRAGA DE Acevedo J. : Novos dados sobre a biologia das especes de Phlebotomus de Lisboa e arredores. Ann. Inst. Med. Trop., 1946, 3, 7-20,

Gaud J. : Phlebotomus du Maroc. Bull. Inst. Hyg. Maroc., 1964, 14, 91-1 ro.

Gil Collado J. : Sobre la domesticidad de las especies españolas de Phlebotomus. Bol. Hist. Nat., I93I, 3I, 505-507.

Gil Collado J. : Phlébotomes et Leishmanioses en Espagne. In : Écologie des Leishmanioses. Montpellier, 18-24 août 1974. Colloques Internationaux du CNRS, 1977, n 239, I77-190.

Guevara Benitez D. C., Ubeda Ontiveros J. M., Morillas Marguez F. : Phlebotominae de la provincia de Granada : estudio de poblaciones. Rev. Iber. Parasitol., 1978, 38, 813-839.

Hourn R. : Nouvelles localisations de Phlébotomes en Espagne. Ann. Parasitol. Hum. Comp., I $965,40,34 \mathrm{I}-363$.

Lewis D. J. : A taxonomic review of the genus Phlebotomus (Psychodidae). Bull. Brit. Museum (Natural History), I982, 45, 121-209.

Maroli M., Bettini S. : Leishmaniasis in Tuscany (Italy). (I). An investigation on phlebotomine sandflies in Grosseto Province. Trans. Roy. Soc. Trop. Med. Hyg., I977, 71, 31 5-321. 
Martinez Ortega E., Ward R. D., Martin Luengo F. : Introducción al estudio de Phlebotomos en Alicante y Murcia (Diptera, Phlebotomidae). Rev. Iber. Parasitol. (Vol. Extraordinaire II Conferencia Mediterránea de Parasitología), I982, 46I-472.

Morillas Marquez F., Guevara Benitez D. C., Gil Collado J., Ubeda Ontiveros J. M. : Presencia en España de Phlebotomus (Larroussius) longicuspis (Nitzulescu, I93o) (Diptera, Phlebotomidae). Rev. Iber. Parasitol. (Vol. Extraordinaire II Conferencia Mediterránea de Parasitología), I982, I9I-196.

Morillas Marquez F., Ubeda Ontiveros J. M., Guevara Benitez D. C., Gonzalez Castro J. : Confirmación de la existencia en España de Phlebotomus (Paraphlebotomus) chabaudi Croset Abonnenc et Rioux, I970 (Diptera, Phlebotomidae). Rev. Iber. Parasitol., I982, 42, 345-346.

Najera Angulo L. : Observaciones sobre los Phlebotomus recogidos en Sigüenza. Actas primer Congreso Nacional de Sanidad, 1935, 4, 305-316.

Najera Angulo L. : Distribución geográfica de Phlebotomus en España y datos relativos a unas 50 localidades nuevas. Rev. Sanid. Hig. Públ., I940, 14, 290-304.

Pires C. A. : Contribuçao ao conhecimento da distribuçao e biologia das flebótomos em Portugal (Diptera, Psychodidae). Bol. Soc. Portuguesa Ciencias Naturales, 1979, 19, 197-210.

Pittaluga G, Buen S. DE : Especies españolas del género Phlebotomus. Bol. Real Soc. Española Historia Natural, I918, $18,377-385$.

Rioux J. A., Golvan Y., Croset H., Houin R., Juminer B., Bain O., Tour S. : Écologie des Leishmanioses dans le sud de la France. I. Les Phlébotomes. Échantillonnage, Éthologie. Ann. Parasitol. Hum. Comp., 1967, 42, 56I-603.

Rioux J. A., Golvan Y., Croset H., Tour S., Hourn R., Abonnenc E., Petitdidier M., Vollhardt Y., Dedet J. P., Albaret J. L., Lanotte G., Quilici M. : Épidémiologie des Leishmanioses dans le sud de la France. Monographies INSERM, n० 37 , Paris, 1969, 2r6 p.

Rioux J. A., Croser H., LÉger N. : Présence en Espagne de Phlebotomus alexandri Sinton I928 (Diptera, Psychodidae). Ann. Parasitol. Hum. Comp., 1974, 49, 126-128.

Rioux J. A., Croset H., LÉger N. : Présence en Espagne de Phlebotomus chabaudi Croset, Abonnenc et Rioux, I970 (Diptera, Psychodidae). Ann. Parasitol. Hum. Comp., 1974, 49, 505-507.

Vives Sabater J. : Biología de las especies de Phlebotomus hallados en la provincia de Barcelona. Rev. Iber. Parasitol., I954, I4, 397-407.

Zariguiey Alvarez R. : Contribution à l'étude de la femelle de Phlebotomus aviasi. Ann. Parasitol. Hum. Comp., I937, Is, 409-418.

Zariouiey Alvarez R. : Contribución al conocimiento de los Phlebotomus en España. Graellsia, I944, $I, 15-20$. 